\title{
Advanced And Economical Android Application Based Attendance System
}

Siddharth Pandey ${ }^{1}$, Anurag Singh ${ }^{2}$, Priya Singh ${ }^{3}$ and Harshali Patil ${ }^{4}$

Abstract- This paper discusses about a way to handle attendance problem and suggest a use of an attendance system. Here we are able to track the students attending a particular lecture without actually wasting time taking the attendance. As today the classroom sizes are huge it is a cumbersome task to keep track of students or take care of attendance in classes which can be solved using the technique discussed in this paper.

\section{INTRODUCTION \\ Dissertation Idea:}

A classroom plays a pivotal role for imparting education to the students. With the growing number of classroom sizes comes a problem of attendance. Today many classes have a class sizes ranging in hundreds of students all eager to gain knowledge. With this there is an increase in time required to take attendance for which everyone has to wait and the precious time for learning is cut short. There have been many ways developed to tackle this issue but they require use of advanced technology and extra hardware costs or they are prone to errors due to high complexity. This paper presents an attendance system which is easier to use and less prone to error and makes use of technologies already existing in institutes

An attendance system should be easy to use by the teachers and students alike. It should not require additional hardware or be incur additional costs. The attendance system must be less prone to errors or technological failures and should be robust. Digitizing the attendance system allows us to not only calculate the attendance faster but also helps us to track the students who have missed a particular lecture and allow them to know what they have missed. It will also help the teacher to track down students who have missed important lectures and invite them for a

${ }^{1}$ Dept. Of Computer Engineering Thakur College of Engineering and Technology, Mumbai, India

${ }^{2}$ Dept. Of Computer Engineering Thakur College of Engineering and Technology, Mumbai, India

${ }^{3}$ Dept. Of Computer Engineering Thakur College of Engineering and Technology, Mumbai, India

${ }^{4}$ Dept. Of Computer Engineering Thakur College of Engineering and Technology, Mumbai, India 
cover up session for the missed lectures. The teacher can also contact students directly who have missed many lectures and have low attendance.

\section{LITERATURE SURVEY:}

In [1] auth. Seema Rao proposes a fingerprint identification system to mark attendance. Here the author first stores the current student fingerprint features using ridges, arch, loops and whorls using feature extraction and stores it in database. Then when students enter the classroom they scan their thumb on the fingerprint scanner which in turn matches the data stored in the database and marks the matched student as present in attendance. The feature extraction again takes place during attendance marking and they are compared with that of those stored in database. When a match is found the student is marked present for that lecture.

The authors in [2] have implemented a login system by which a student can login to a server and mark his or her attendance. They have stored all information on a database and have a login system. After logging into the logging system the student can mark his attendance anytime and after which the lecturer will send the attendance to the server system using GPRS. The lecturer can enrol new students or delete existing ones, modify information about existing students.

In [3] the authors Mr. Gautam Shanbhag and Mr. Hussain Jivani use android system as well as a biometric system for authentication. Here the professor logs into the system using the provided username and password. After logging into the system he creates a lecture schedule by providing all the details of the lecture that include timing, duration of lecture, subject, topic in the subject and location. The students then enter the class and login into server give their attendance. After that they punch their fingerprint in portable fingerprint scanner and if they match their attendance is confirmed.

In [4] the authors S.P. Avinaash Ram and J. Albert Mayan have developed an attendance system for employees. Here they track every employee within premise using the GPS on the employee device. The admin has the IMEI number of the devices of the employee. The employee will register attendance using the smart phone. He can be tracked using the GPS and other staff can also know the location of other people using their GPS. Once the employee leaves the premise he is logged out and the information is stored.

In [5] the authors Unnati A. Patel and Dr. Swaminarayan Priya R. use RFID and facial recognition to avoid errors or prevent loopholes in their project. But by using RFID requires an extra hardware cost and all phones do not have NFC, as well as facial recognition is not very reliable. It may happen that the facial recognition system may fail to identify a legit user of the attendance system.

In [6] the authors Tabassam Nawaz, Saim Pervaiz, Arash Korrani,Azhar-ud-din use fingerprint mechanism to authenticate the students with constraints on time to mark the attendance. But as we know not all students can simultaneously, which would be a problem for a class with huge class size. As well there needs to be a fingerprint sensor deployment and the fingerprint sensor cannot be fully correct. Furthermore, they do not use any other technique to mark the attendance which would make it difficult for a student to mark his attendance when his fingerprint would not match due to some problem.

\section{PROBLEM DEFINITION AND SCOPE Problem Definition:}

All current attendance systems require some exclusive hardware technology which adds to the cost of the system. The attendance systems also require technology that is fault prone. The biometric system as used in [1], [3], [4],[5] and [6] requires usage of a fingerprint scanner that 
has till date not been totally accurate. A person may have to try multiple attempts to get it right. Furthermore, the attendance does not go on parallel for everyone, all the students have to get the biometric check done one after another making others wait. In [5] and [6] there is no robust mechanism that would allow students facing problem while entering their fingerprint to mark their attendance. They cannot mark their attendance if they are not able to use the system. In [2] the proposed method does not use much of technology and allows logging into server using GPRS, which a student can log onto from outside the campus and mark his attendance without actually attending the lecture. In [4] there is tracking involved using GPS which may use a lot of battery from the device and furthermore it makes use of biometric fingerprint for attendance which till date is not totally accurate and error free. So we can see that most techniques require extra hardware that would incur or raise the cost of the whole system. These hardware devices are not ubiquitous in institutes and they would have to be bought specifically for making the attendance system.

\section{Scope:}

- This system uses only basic equipments already present and ubiquitous in all educational and professions requiring attendance systems. No additional resources are required for the implementation of the system hence reduced cost.

- This system cannot be fooled easily as one has to be in the premise to register the attendance. Hence the system is robust and secure.

- This system is flexible and can easily be integrated with existing systems and can also be easily upgraded to match more safety requirements. For example biometrics like fingerprint scanners which are catching up in mobile devices can be easily be implemented in this system. Hence the new system is flexible.

- This implementation will reduce manual work and save time.

- This implementation will use the current and updated technology like android.

- In case of device failure the updation can be done manually too making this system robust.

\section{Objectives:}

- Economical equipment: to use equipment that is easily and economically available.

- Secure and foolproof: one would have to be on the campus to register attendance which cannot be fooled easily.

- Easy to implement: this system is user friendly as it is an application on mobile device.

- Flexible: it can make use of additional authentication devices used on the premise and can easily be integrated with the same.

- Robust: on failure of particular devices of the system, the data can be recorded manually and later fed into the system.

\section{SYSTEM DESIGN}

\section{Enhanced Attendance System:}

Considering the need to reduce manual intervention and automate the traditional processes along with keeping in mind the upcoming technologies we formulated a new concept that reduces manual work by a huge amount. Attendance has been one of the most important and the very first need in every classroom. Considering the amount of time and energy wasted in this process has led us to develop a much more robust, clean and accurate concept of automated attendance mechanism. 
The methodology works on the latest technology, major part of which will be operating on the most used operating system, android. The concept comprises of two different applications, one for the students and other for the professors. Being user friendly the application guides the user through basic steps where attendance can be recorded and stored in the database.

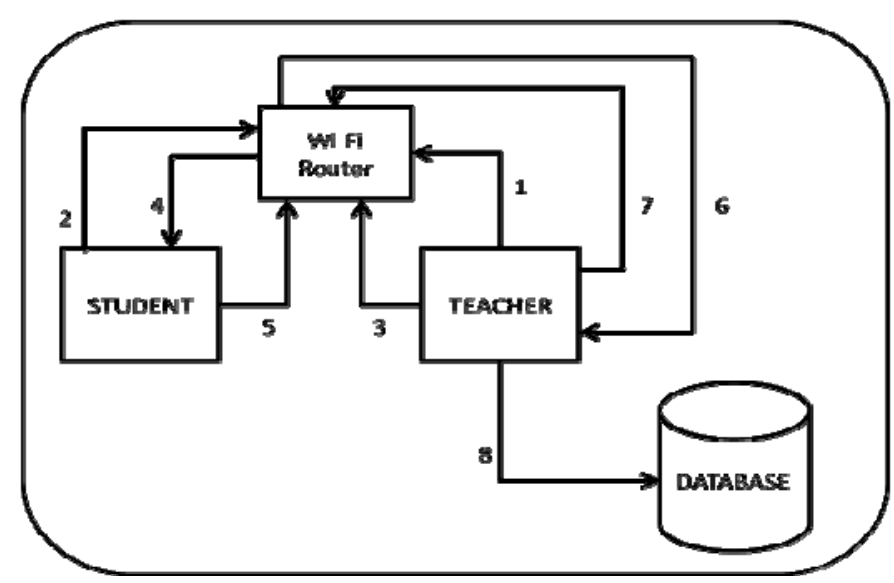

Fig 1. Framework of the proposed approach

Figure 1, depicts the entire framework of the proposed approach. Each of the steps have been explained below.

- Step 1: The teacher initiates the lecture by logging into the VPN and entering all the details of the lecture that include subject, timing, duration and location of the lecture.

- Step 2: The students log into the VPN using the session key announced by the teacher for the session. Once logged in the student will wait for the teacher to send an OTP for verification.

- Step 3: The teacher sends an OTP to VPN which broadcasts it to all the students.

- Step 4: The students receive the OTP from the Wi-Fi VPN.

- Step 5: The students then enter OTP in the classroom session from their app and confirm attendance.(Only the students inside the VPN that is in classroom connected to WIFI can receive and send the OTP).

- Step 6: The attendance is then passed to the teacher.

- Step 7: The teacher ends the current session preventing any other students to login to the same session.

- Step 8: The teacher after completing the attendance sends the attendance information for storage to the external server after finishing the session.

The process involves setting up a VPN connection in individual classrooms using Wi-Fi routers. The Professor and the students connect to the network using the VPN. This step just allows the users to connect to the network but not actually mark their attendance. Use of VPN assures that the network is confined to a single classroom thus preventing any student from marking the attendance from an external network. A VPN acts a temporary session which is activated using a 
session code and once the process of marking attendance by the students is complete the professor can end the session which would not allow anymore entries after that. Once the VPN has been setup and all the users have been connected the professors triggers a process which sends One Time Password(OTP) to the students connected on to the network. The OTP acts as a second layer of authentication after the VPN which will ensure a balance in the number of entries and the number of students.

As soon as students pass through the second layer of authentication they are directed to a data page where they have to select the appropriate details for the session. The data to be selected by students includes the academic year, respective branch, subject name and date. Once the data has been fed the last thing that student has to do is mark the attendance by pressing the 'PUSH ATTENDANCE' button which records their attendance for the session. The professor has to set up a similar data as that entered by the students in order to avoid any discrepancies. Once the process of marking attendance has been completed the professor can end the session by tapping on the 'END SESSION' button which closes the session and pushes all the data to the database.

Students are allowed to mark the attendance only once every session, however considering the human tendency to commit mistake the application makes sure that multiple entries for a single registered student is stored as a single entry thus reducing the overhead of inconsistent data on the database.

The expected outcome of the proposed methodology has been illustrated below.

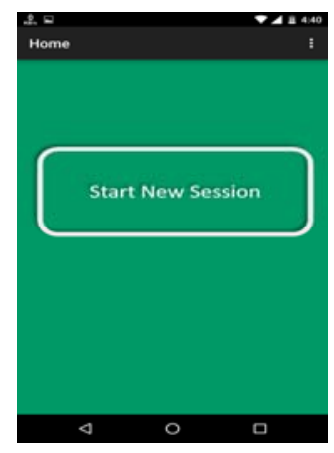

Fig 2. Teacher initiates a new session
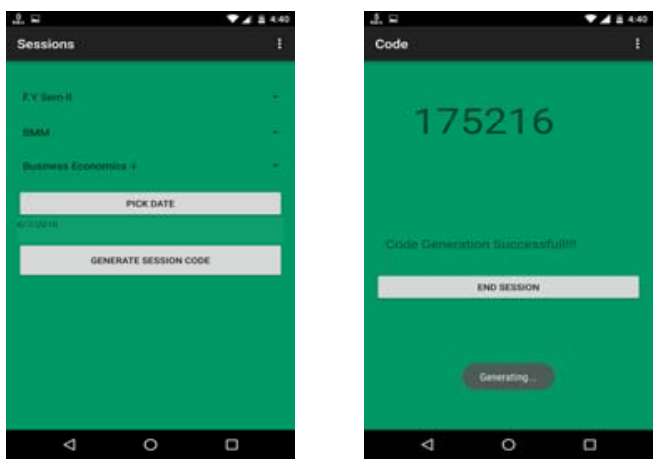

Fig 3. Teacher enters details of the lecture session and gets a generated session key 

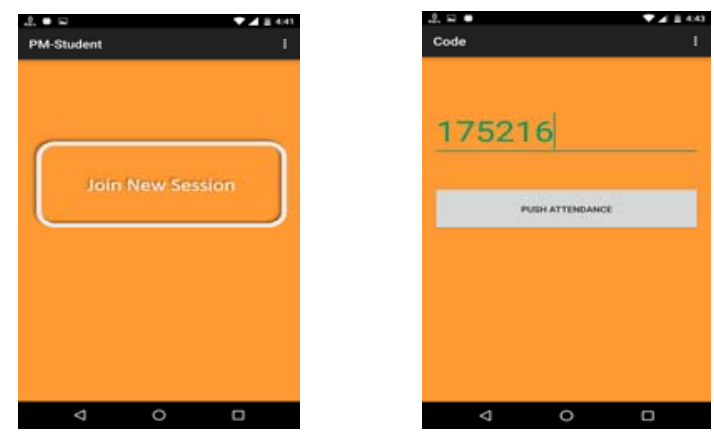

Fig 4. Students enter the new session by entering the session key shared by the Teacher
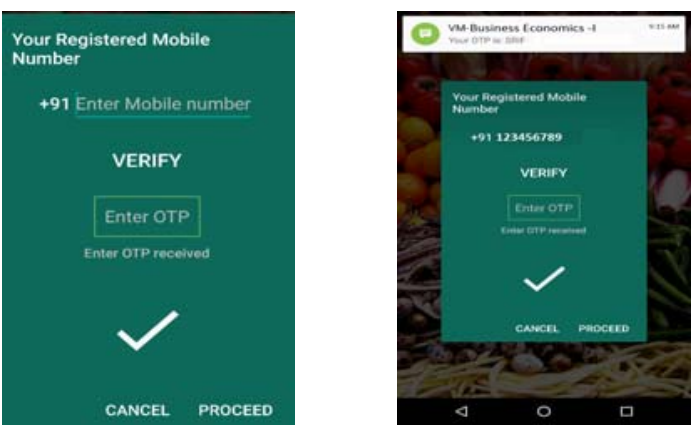

Fig 5. After connecting to session student enters his mobile number(ID) to receive the success

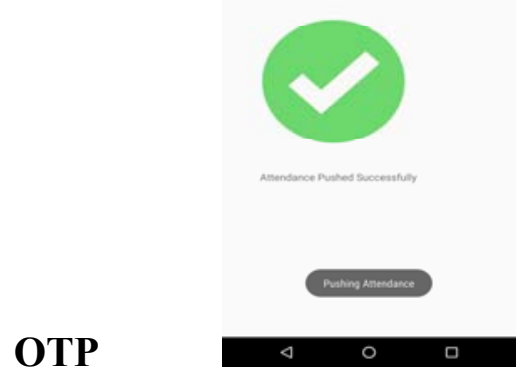
:

Fig 6. Student after entering OTP has his attendance marked

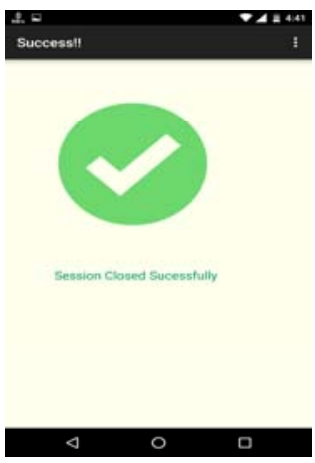

Fig 7. The Teacher ends the session and attendance data is recorded on the server. 


\section{EASE OF USE}

An innovation demands functionality, performance and stability, but all of these are available at a cost of complex technology, complicated user interfaces and bulky customizations. Our app is concerned with the usability and performance delivery at the same time for different types of users. A user may be a day to day user of smart technology or a naive one who has been just introduced to the basics of smart era, the app provides the following features which add more to its performance.

\section{A. Easy Setup and user friendly interface}

Android devices have really been handy for today's generation which follows the three simples steps Download-Install-Use. Keeping in mind the needs and also considering the simplicity, the application is provided in the form of an Android application package file (APK) which the user can simply install. The application can be installed on android devices having an OS version of Gingerbread (v2.3.7) and above. The setup requires just a tap and the application is up and running after which a user can log-in and start using the application. Once the setup is completed , the application acts as a self guiding tool for the users, which is hassle free and straight to the point.

\section{B. Less Prone to Mistakes}

Humans have the tendency to make mistakes unintentionally, however technology has been smart enough to correct those mistakes or even prevent them from occurring at the very first place. Attendance in class rooms have been a major part of the curriculum for the students and the professors, its not always possible to be accurate while maintaining records, thus this is where the application saves the day by reducing manual work and mistakes by a greater margin.

\section{Robust Nature}

It is not always possible for human beings to be in touch with technology, which leaves them with no option but to rely on others for the same. For example, a student forgets his android device without which he won't be able to mark his attendance and thus he has to approach someone for help. Since the application considers security as its basic principle, it won't allow the student to take help form another student but from a person who has much higher authority. Student can approach the professor in such situations, the professor holds the authority and access to student's information and thus will be the perfect choice for help in such scenarios. This flexibility is provided by the application as a part of its robust nature which ensures undisrupted continuity of the workflow.

\section{Regular Tracking and Status Updates}

The application plays an effective role in letting the user know his status regarding the attendance. A user can get his current status any time however the application provides a privilege which generates a weekly report for the user by tracking his attendance. If the user, i.e. the student in this case fails to make it to the safe limit a status message will be displayed a warning to the user, based on which the student can compensate in the cover up lectures which also come as part of the status updates. A similar mechanism will be deployed for the professors which could help them to trace the defaulters on weekly basis and thus would help them to set a cover up lecture for the same. Thus in this way the students and professors will be aware of student's status and regularity in the classes. 


\section{E. Cost Effective and Easy Availability}

New technology requires more hardware which adds to more cost and makes it cumbersome to handle such devices and the system. Our methodology makes use of VPN which can be setup using simple devices like smart phone, laptop hotspots or Wi-Fi router which are ubiquitous in educational institutes. Thus allowing our system setup to be easier and economical.

\section{CONCLUSIONS}

Using the proposed methodology we are able to doubly verify the student and be able to mark his attendance. A student cannot mark his attendance if not present in the classroom where the VPN exists and where the teacher shares the session code. The OTP serves to verify the student and so no external person can be marked for attendance in this system. Here we do not use any external hardware or tools that may increase the cost of implementation. This system can be implemented using the basic hardware present in most institutes. In future this system can be utilised to secure uses data on cloud and also can be useful foe securing large amount of data.

\section{FUTURE SCOPE}

The teacher can use a QR code which can be scanned by everyone in classroom. The teacher may either show the QR code on projector or pass a paper containing the QR code which when scanned using the app will automatically add the attendance of the corresponding student, instead of the OTP. The students cannot send a picture of QR code to someone else as the QR code has to be scanned using the app. This removes the burden of entering the OTP or having a VPN. Today many phones have a fingerprint scanner and after sometime almost all android devices will have a fingerprint scanner, which may be implemented in the app for better security but may not be a necessary feature

\section{REFERENCES}

[1] Seema Rao, Prof.K.J.Satoa, An Attendance Monitoring System Using Biometrics Authentication,in International Journal of Advanced Research in Computer Science and Software Engineering vol. 3, Issue 4,2013.

[2] Freya. J. Vora, Pooja. L. Yadav, Rhea. P. Rai, Nikita. M. Yadav, "Android Based Mobile Attendance System" in International Journal of Advanced Research in Computer Science and Software Engineering vol. 6, Issue $2,2016$.

[3] Mr.Gautam Shanbhag, Mr. Hussain Jivani, Mr. Sushil Shahi,"Mobile Based Attendance Marking System Using Android And Biometrics" in International Journal for Innovative Research in Science \& Technology (IJIRST), vol.1, Issue 1, 2014.

[4] S.P. Avinaash Ram and J. Albert Mayan ,"Mobile Attendance Management And Employee Registration", ARPN Journal of Engineering and Applied Sciences, vol. 10 , No. 8 ,2016.

[5] Unnati A. Patel, Dr. Swaminarayan Priya R.," Development of a Student Attendance Management System Using RFID and Face Recognition: A Review ", International Journal of Advance Research in Computer Science and Management Studies, vol. 2, Issue 8,August 2014.

[6] Tabassam Nawaz, Saim Pervaiz, Arash Korrani,Azhar-ud-din," Development of Academic Attendence Monitoring System Using Fingerprint Identification ",International Journal of Computer Science and Network Security (IJCSNS), vol. 9, No.5, May 2009. 